\title{
Temperature Insensitivity of the Flow Stress in Body-centered Cubic Micropillar Crystals
}

\author{
Yinan Cui*,Giacomo Po, and Nasr Ghoniem \\ Mechanical and Aerospace Engineering Department,University of California at Los Angeles \\ (UCLA), Los Angeles, CA 90095, U.S.A.
}

\begin{abstract}
Plasticity of body-centered cubic (bcc) crystals is known to have a strong dependence on temperature, as a direct consequence of the thermally-activated process of kink pair nucleation and migration with a high energy (Peierls) barrier. Here we demonstrate that, in the sub-micron size scale, such strong temperature dependence of the flow stress must disappear. We explore the flow stress and hardening behavior of micro-pillar sizes in the range 200-2000 $\mathrm{nm}$ at temperatures of 150-900 K. Discrete Dislocation Dynamics (DDD) simulations reveal that the weak temperature sensitivity can be rationalized in terms of the weak role of screw dislocations in controlling plasticity; unique to small crystals of finite size. It is shown that finite, sub-micron samples have limited ability to store screw dislocations. The necessity of applying high stress in sub-micron crystals is demonstrated to greatly enhance the mobility of screw dislocations, rendering it close to that of edge dislocations. This leads to a transition of the dislocation mobility mechanism from being thermal-activated kink dominated to being phonon-drag dominated. Thus, the flow stress gradually becomes not governed by the mobility of screw dislocations (as determined by the critical resolved shear stress), giving rise to the weak temperature sensitivity of the flow stress. A dislocation mechanism map in the temperature-size space is proposed to further illustrate this phenomenon in tungsten micropillars.
\end{abstract}

Email address: cuiyinan@g.ucla.edu (Y.N. Cui) gpo@ucla.edu (G. Po), ghoniem@ucla.edu (N.M. Ghoniem) ()

Preprint submitted to Acta Materialia

January 28, 2016

(C) 2016. This manuscript version is made available under the Elsevier user license http://www.elsevier.com/open-access/userlicense/1.0/ 
Keywords: Tungsten, Micro-pillar, Flow stress, Temperature effect, Size effect

\section{Introduction}

A number of experiments have demonstrated that the flow stress in all bodycentered cubic (bcc) crystals decreases sharply as the test temperature is increased. This behavior is observed up to a critical temperature, above which the flow stress becomes athermal[1-3]. This phenomenon is generally attributed to a fundamental mechanism that controls dislocation motion in bcc crystals. Kink pairs, at temperatures below a critical value, must be nucleated on the nonplanar core structure of screw dislocations by overcoming an energy (Peierls) barrier. Subsequent migration of nucleated kinks leads to overall dislocation motion. In addition to the key influence of the particular symmetric bcc crystal structure, several other factors have been found to affect the extent of the strong temperature dependence. Crystal purity, the polycrystalline nature of large-size samples, and the loading orientation have been shown to influence the temperature dependence of the flow stress. Tungsten, as a prototypical bcc metal, offers great potential for ultra-high temperature applications, such as in magnetic fusion energy devices, plasma-facing components[4], and space electric propulsion. As a representative bcc crystal, the plastic behavior of bulk tungsten is known to be strongly temperature sensitive, and is thus a good candidate for understanding the temperature dependence of the flow stress at sub-micron sizes.

Recent studies on the plasticity of bcc crystals in the micro- and nanometer size range revealed that the limited sample size usually contributes to new features of the plastic behavior. For example, plastic flow becomes size dependent, and the stress-strain relationship exhibits discrete strain bursts instead of the smooth and continuous plastic flow, characteristic of ploycrytals[5-7]. An interesting question is whether the temperature will still have the same strong influence on the flow stress in small volumes, as is the case in conventionally large specimens. Coupling effects between temperature and sample size on the 
plastic deformation and the underlying dislocation mechanisms are thus of great interest. Investigations on these problems will shed light not only on our understanding of submicron plasticity in bcc crystals, but will also provide guidance on improving the mechanical properties of thermomechanical devices through control of the crystal size and structure.

Due to the particular symmetry of the bcc crystal structure and the complex dislocation core transformations induced by the applied stress[8, 9], the plastic behavior of bcc metals is much more complicated than that of fcc metals. Thus, the deformation mechanisms of bcc crystals are still relatively poorly understood $[10,11]$. To reveal the plasticity behavior of sub-micron bcc crystals, micropillar compression and tension tests are recently widely used. The focus 40 of such experiments is generally on the size effect, the strain burst behavior, the anomalous slip, the comparison with fcc crystals, and the possibility of mechanical annealing $[6,7,12-14]$. Some researchers have also investigated the surface orientation effect[15], and the hydrogen embrittlement behavior[16]. Nevertheless, the vast majority of experimental investigations on submicron bcc crystals 45 are carried out at room temperature. Only recently, Schneider et al. compared the size effect of bcc Mo micropillars at room temperature to that at elevated temperatures[17]. Although the influence of external size on the temperature dependence of plasticity is not explicitly studied in that work, the limited experimental results (see Fig. 3(a) in [17]) still imply the interesting trend that 50 the temperature sensitivity seems to decrease with reduction of the sample size. Till now, little attempt was made to systematically clarify the coupled effects of sample size and temperature on the plasticity of bcc crystals.

The method of Discrete Dislocation Dynamics (DDD) has proven to be a powerful approach for revealing the deformation mechanisms and the mechan55 ical response of submiron crystals[18-22]. It offers the unique advantage that the conditions of the simulation can be controlled very precisely, and can be directly compared to experiments at the same size and length scale. By incorporating the insights gained from atomistic simulations, the specific features of bcc plastic flow have been successfully captured with DDD simulations, such as 
the non-Schmid effect, the twinning/anti-twinning and the tension/compression asymmetries, etc[23-26]. Therefore, the size effect on the temperature dependent flow stress in small scale bcc crystals is explored in this work using our recently improved atomistically informed DDD model[27].

The paper is organized as follows. In the next section, we provide a brief description of our DDD simulation method. In section 3, the computer simulation results are presented and compared with available experimental data. Then, the combined influence of sample size and temperature on the flow stress is presented. The reason why the small size of micro pillars influences the temperature dependence of the flow stress is analyzed in section 4. A preliminary dislocation mechanism transition map, influenced by sample size and temperature is then proposed in section 5. Concluding remarks are finally given in section 6 .

\section{Computer simulation setup}

The DDD method used here has been described in detail in our previous papers $[19,28,29]$. In this model, dislocation loops of arbitrary shape are split into contiguous curved segments. These, in turn, are discretized into a succession of parametrized cubic splines containing a set of Gauss integration points. The elastic field (stress, strain, and displacements) are all obtained by a fast sum method [28], assuming that dislocations are in an infinite medium. The topology of all dislocations updates every time-step to deal with dislocation junction formation, annihilation, and the occurrence of cross slip. The boundary conditions and image force effects of finite geometry of any shape are considered by utilizing the superposition principle of Eshelby [30]. The finite element method makes boundary corrections to the elastic filed of dislocations in an infinite medium

${ }_{85}[19]$. The resulting computational method is implemented in the open-source $\mathrm{C}++$ computer program: Mechanics Of Defect Evolution Library (MODEL) $[31]$.

An atomistically-informed dislocation mobility law for bcc crystals is used 
here [27]. Since dislocation mobility is significantly different for screw and edge dislocations, the velocity $v_{m}$ of an arbitrary mixed dislocation segment is calculated by separating the edge and screw components, and the total velocity is written as,

$$
v_{m}=v_{e} \cdot w(\theta)+v_{s} \cdot(1-w(\theta))
$$

where $\theta$ is the angle between the dislocation tangent direction and the Burgers vector direction. $w(\theta)$ is dimensionless weigh function with properties $w(0)=0$, and $w(\pi / 2)=1$. Here, $w(\theta)$ is taken as $\sin ^{2} \theta[27]$. Note that the choice of $w(\theta)$ does not qualitatively affect the results presented in the article. The velocity of the edge component, $v_{e}$, is determined by the total force acting on it divided by the viscous drag coefficient.

$$
v_{e}=\frac{\tau_{r s s} b}{A_{e} T}
$$

where $\tau_{r s s}$ is the resolved shear stress, which includes the Peach-Koehler (PK) force due to the applied stress and other defects, the self-force, as well as the boundary image force. $A_{e} T$ is the drag coefficient of an edge dislocation, $T$ is the test temperature, and $A_{e}$ is a phonon drag constant. For screw dislocations, kink nucleation and motion is taken into account through an Arrhenius-type equation of the form,

$$
v_{s}=\frac{\tau_{r s s} b}{A_{s} T} \exp \left(-\frac{\Delta G}{k_{b} T}\right)
$$

where the Gibbs free energy for kink pair nucleation, $\Delta G$, is given by the phenomenological relations [32],

$$
\begin{aligned}
\Delta G(\boldsymbol{\sigma}, T) & =\Delta H(\boldsymbol{\sigma})-T \Delta S \\
& =\Delta H_{0}\left[\left(1-\left(\frac{\tau^{\alpha}}{\tau_{P}}\right)^{p}\right)^{q}-\frac{T}{T_{c}}\right]
\end{aligned}
$$

90

where $v_{s}$ is the screw dislocation velocity, $T_{c}$ is a critical temperature, above which motion is no longer controlled by kink pairs and phonon drag dominates, $k_{b}$ is Boltzmann's constant, $\Delta H_{0}$ is the activation enthalpy at zero stress. Also, $p$ and $q$ are two constants reflecting the shape of the Peierls potential, which can 
be determined by atomistic simulations and experimental data, $\tau_{P}$ is Peierls

stress, $\tau^{\alpha}$ is an effective resolved shear stress (rss), which takes non-Schmid effects into account[33], and is given by:

$$
\begin{array}{r}
\tau^{\alpha}=\boldsymbol{\sigma}: \mathbf{m}^{\alpha} \otimes \mathbf{n}^{\alpha}+a_{1} \boldsymbol{\sigma}: \mathbf{m}^{\alpha} \otimes \mathbf{n}_{1}^{\alpha}+ \\
a_{2} \boldsymbol{\sigma}:\left(\mathbf{n}^{\alpha} \times \mathbf{m}^{\alpha}\right) \otimes \mathbf{n}^{\alpha}+a_{3} \boldsymbol{\sigma}:\left(\mathbf{n}_{1}^{\alpha} \times \mathbf{m}^{\alpha}\right) \otimes \mathbf{n}_{1}^{\alpha}
\end{array}
$$

where $\boldsymbol{\sigma}$ is the total stress tensor, including the contribution from the PeachKoehler force, dislocation interaction stress and the image force. $\mathbf{n}^{\alpha}$ is the slip plane normal vector, $\mathbf{m}^{\alpha}$ is the slip direction, $\mathbf{n}_{1}^{\alpha}$ is the unit normal to the $\{110\}$ plane in the $\mathbf{m}^{\alpha}$-zone that makes a $60^{\circ}$ angle with the slip plane [33]. The second term on the right hand of Eq. 6 represents the contribution of twinning/antitwinning asymmetry and the third and fourth terms account for the loading compression/tension asymmetry. $a_{1}, a_{2}$ and $a_{3}$ are non-Schmid material parameters that determine the effects of each non-Schmid stress component on dislocation glide. Their values are determined by atomistic simulations and experiments [27].

In this study, a series of DDD simulations of tension test are performed on W single crystal micropillars with diameters in the range 200-2000 nm. For all studied samples, the aspect ratio of height $h$ to diameter $d$ is fixed to 2. Tensile loading along the [001] direction is applied in order to achieve multi-slip conditions. Only glide on $\{110\}$ slip planes is considered. The sample temperature is varied in the range 150-900 K. W is approximated to be elastically isotropic, and the following materials properties are used: shear modulus $\mu=161 \mathrm{GPa}$, Poisson's ratio $\nu=0.28, \mathrm{p}=0.86, \mathrm{q}=1.69, a_{1}=0.938, a_{2}=0.71, a_{3}=4.43$, $\Delta H_{0}=1.63 \mathrm{ev}[34,35], \tau_{P}=960 \mathrm{MPa}$, which is obtained by extrapolating the experimental data for high purity $\mathrm{W}$ single crystal[36], $A_{e}=3.3 \times 10^{-7}(\mathrm{~Pa} \cdot \mathrm{s} / \mathrm{K})$, and critical temperature $T_{c}=800 \mathrm{~K}$.

Initial equilibrium dislocation configurations are generated through the relaxation of randomly distributed dislocation loops without external loading until further dislocation activity is not noticeable. Dislocation junctions emerge as 


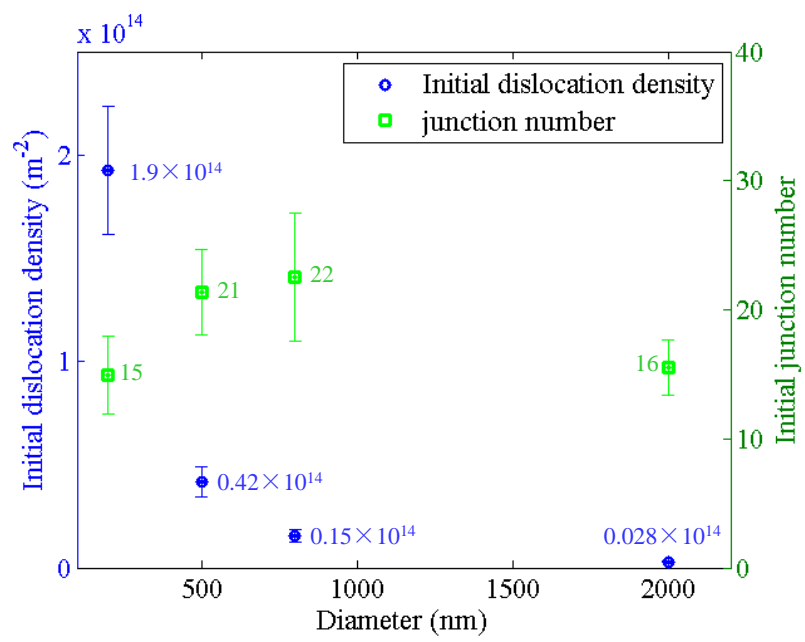

Figure 1: The initial dislocation density (labeled near the point) and initial number of dislocation junction after relaxation

a natural outcome of dislocation interactions without artificially enforcing pinning points. The initial dislocation density is higher for pillars with smaller diameter. This is designed in order to generate comparable number of initial dislocation junctions in small pillars with that in large pillars. For each sample size, multiple different initial dislocation configurations are used in DDD simulation. Quantitatively, after relaxation, the initial dislocation density and the initial number of dislocation junctions used in the simulations are plotted in Fig. 1, where the points are the averaged values over multiple simulations for the pillar with each diameter, and the error bar is the standard deviation. The applied strain rate was equal to $10^{4} s^{-1}$. Even though it is higher than the experimental value, the steady-state flow stresses are found not to be sensitive to the strain rate [14], which is also verified by comparing our simulation results with experimental data as will be discussed below.

\section{Temperature and size dependent flow stress}

Typical tension test stress-strain curves for various sample sizes of $\mathrm{W}$ micropillars at different temperatures are plotted in Fig. 2. For each size, the re- 
(a)

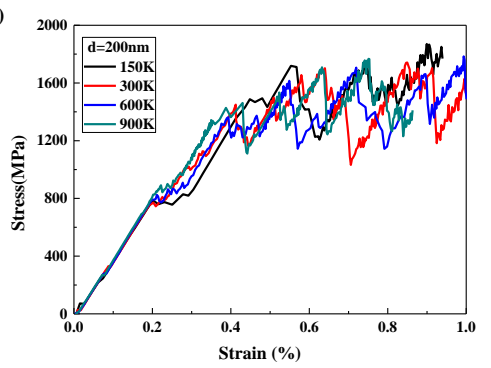

(c)

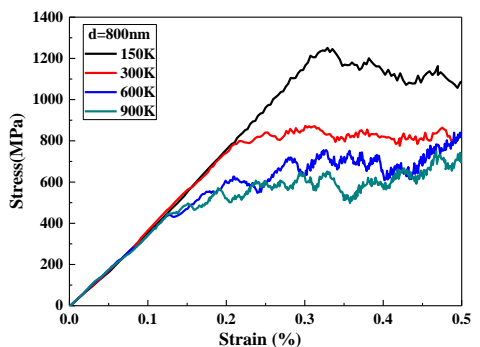

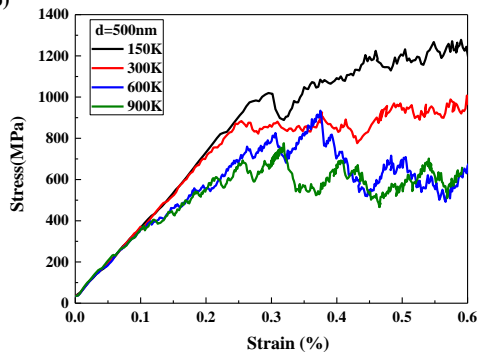

(d)

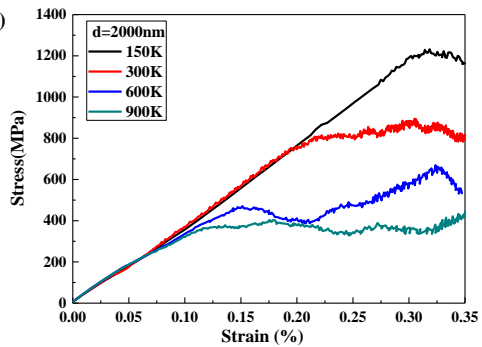

Figure 2: Stress-strain curves for micropillar tension test under different temperatures with diameter (a) $200 \mathrm{~nm}$, (b) $500 \mathrm{~nm}$, (c) $800 \mathrm{~nm}$ and (d) $2000 \mathrm{~nm}$

sults corresponding to the same initial dislocation configuration are presented at different temperatures to avoid the influence of the initial dislocation structure on the results. It is clear from the figure that, regardless of test temperature, all pillars with diameter smaller than $2000 \mathrm{~nm}$ exhibit elastic loading followed by significant serrated stress oscillations, which is consistent with experimental observations [7]. In addition, for large sample sizes, significant temperature effects of the flow stress are easily observed, similar to the behavior of bulk specimens. Surprisingly, however, this temperature dependency almost vanishes when the sample size is roughly smaller than $200 \mathrm{~nm}$.

Before disclosing the physical origin of this interesting phenomenon, our simulation results are first compared with available experimental results to validate their effectiveness. Since most experimental investigations focus on the size effect on the flow stress at room temperature, the yield strength for micropillar $(\mathrm{d}<1000 \mathrm{~nm})$ at room temperature are first evaluated and compared with experiments[6]. This yield strength is defined as the $0.2 \%$ offset yield point $\sigma_{0.2}$, 


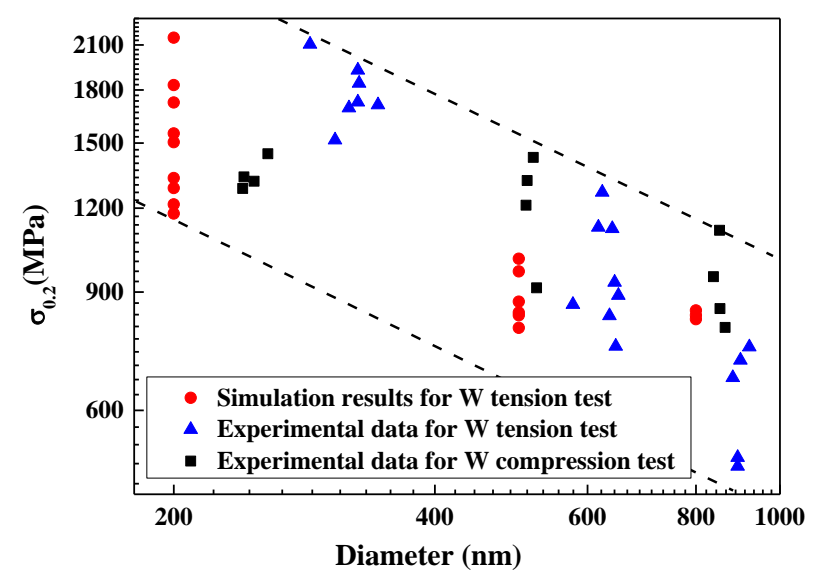

Figure 3: Comparison of the yield strength of $\mathrm{W}$ micropillars with experimental data [6] at room temperature. Note the scatter in the data, which is inherent in the plasticity behavior of small size samples.

as is standard in engineering stress-strain relationships of bulk samples. Fig. 3 compares the calculated $0.2 \%$ offset yield stress and available experimental data at room temperature, showing generally good agreement. Up till now, systematic experimental studies of the plastic deformation of $\mathrm{W}$ micropillars at low and elevated temperatures are not available. However, several experimental studies pointed to the diminished size dependence of the flow stress, as the normalized temperature is decreased[17, 37]. Such inspiring investigations make it possible to reveal the temperature-influenced size effect on the flow stress. This is achieved by directly comparing yield strength data of different BCC metals at room temperature, since the critical temperature is material dependent, and room temperature would correspond to different homologous temperatures. As shown in the logarithmic plot in Fig. 3, the size effect can be expressed as a power law relation, $\sigma_{0.2}=A d^{n}$, where $d$ is the pillar diameter, and $A$ and $n$ are fitting parameters. According to the present computer simulation results, the calculated power law exponent $n$ can be plotted in Fig. 4 as a function of the temperature normalized by the critical temperature. This normalized temperature $T / T_{c}$ actually reflects the extent of how screw dislocations influence 
the plastic behavior. When $T / T_{c}>1$, namely the temperature is higher than the critical temperature, screw dislocations have similar mobility to edge ones. This implies that the dislocation behavior in BCC crystals becomes similar to that of FCC crystals. According to previous studies[5], the power law exponent for FCC crystals was found to be about -0.6 , as indicated by the dotted line in Fig. 4. As expected, the calculated power law exponent at a temperature $900 \mathrm{~K}\left(>T_{c}\right)$ is -0.59 , which is close to -0.6 . By contrast, as $T / T_{C}$ decreases, the absolute value of the power law exponent decreases. Quantitatively, as $T$ changes from $600 \mathrm{~K}$ to $300 \mathrm{~K}$ and further to $150 \mathrm{~K}, n$ varies from -0.51 , to -0.42 and to -0.09 , respectively. This implies that the size effect becomes weaker at lower normalized temperatures. This is consistent with the experimental results of Schneider and coworkers[17, 38, 39], as plotted in Fig. 4. The deviation of the simulation and experimental results may be associated with the exact definition of the yield strength and also variations in the initial dislocation density. Moreover, the experimental results are corresponding to compression test, while the simulation are corresponding to tension test. According to Kim et al.'s work [6], the size effect seems stronger for tension test compared with compression test for $\mathrm{W}$ micropillar at room temperature.

Comparison of the computer simulation results with the experimental data indicates that the simulations are reliable, and that further predictions can be made based on computations alone. In the following, results of simulations will focus on revealing the interplay between sample size and temperature in determining the flow stress and post-yield hardening in bcc micropillars. To quantitatively make this determination, it is valuable to first compare the yield stress at different temperatures for various pillar sizes. Considering that the stress-strain curve for submicron crystals displays strong statistical fluctuations 195 (see Fig. 2), and that the yield stress is greatly influenced by the initial dislocation microstructure, an averaged yield stress is obtained. This is done by simulations of various realizations for each micropillar size and test temperature, and then calculating the $0.2 \%$ offset yield stress corresponding to the averaged stress-strain curve. As shown in Fig. 5, the strong stress oscillations almost 


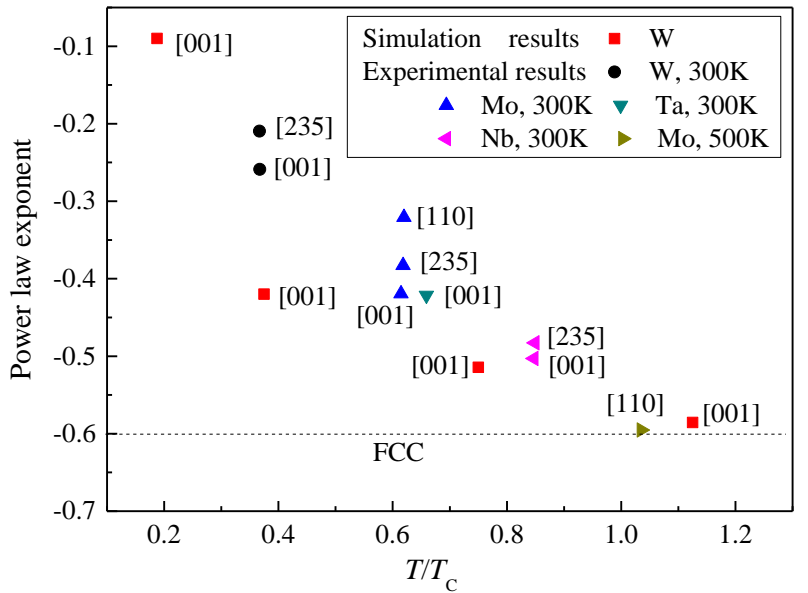

Figure 4: Dependence of the yield strength power law exponent on test temperature. The experimental data are from the work of Schneider et al. [17, 38, 39]. The loading orientation is labeled near the data points

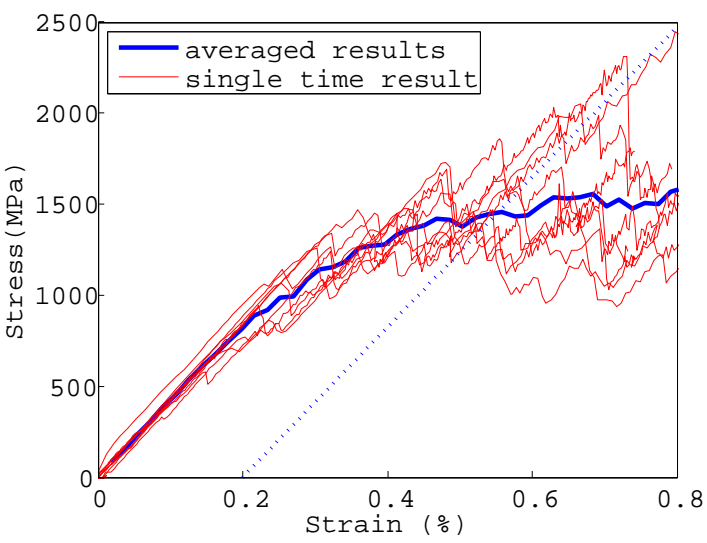

Figure 5: An example showing the method of defining the flow stress of a micropillar with diameter of $200 \mathrm{~nm}$ at $T=300 \mathrm{~K}$. The flow (yield) stress corresponds to the intersection between the dotted line (drawn parallel to the elastic line), and the thick stress-strain curve representing average behavior from several statistical realizations. 


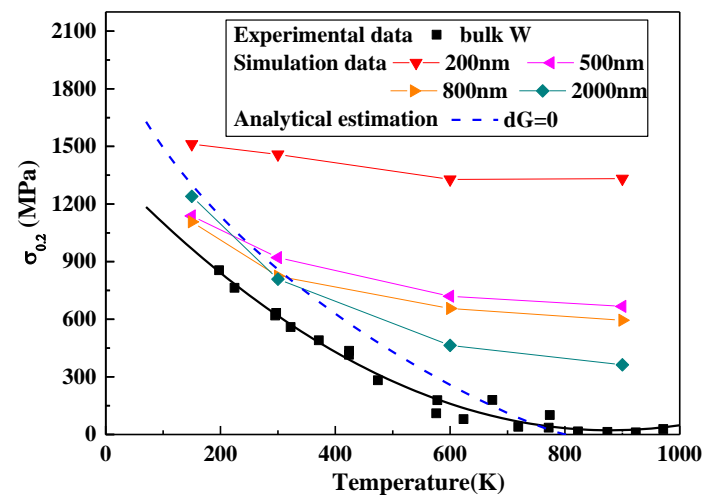

Figure 6: The dependence of the flow (yield) stress on temperature for various sample sizes. Note the insensitivity of the flow stress to test temperature when the sample size is small (e.g. $200 \mathrm{~nm})$.

smooth out into a continuous curve. Since the smaller the pillar size, the more significant the stochasticity is, more simulations are carried out to obtain the averaged data for smaller pillars.

The calculated averaged results are summarized in Fig. 6, together with the $0.2 \%$ offset yield stress obtained experimentally for bulk tungsten [2]. Since it is generally accepted that the flow stress is highly orientation dependent [1], the experimental results also correspond to the [001]-orientation tension test, which is the same as the simulation set-up. Figure 6 clearly illustrates the combined temperature and size effects on the yield stress. As the sample size decreases, the temperature sensitivity of the yield stress becomes weaker. For small pillars, the yield stress almost exhibits no temperature dependence. Note that the flow stress for pillars with diameter $2000 \mathrm{~nm}$ at $150 \mathrm{~K}$ seems somewhat higher. This is on the one hand related to the weak size effect at low temperatures (see Fig.4). On the other hand, a relatively lower initial dislocation density is used when simulating the larger sample (see Fig. 1), in order to generate a comparable number of initial dislocation junctions in pillars with different sizes. This may contribute to the high flow stress. 


\section{Weak role of screw dislocation at small scale}

It is natural to ask what controls the temperature sensitivity. For bulk BCC crystal, the strong temperature sensitivity of flow stress is always attributed to itself through significantly different screw dislocation mobility at different temperatures. Thus, different stress levels are required to maintain the required plastic flow. Based on this opinion, the weak temperature dependence at small scale implies that the role of screw dislocation becomes less pronounced. The

\subsection{Limited screw dislocation storage ability}

Small sample size generally means higher probability of dislocation surface annihilation. In FCC crystal, this can contribute to significant decrease of dislocation density, even causing mechanical annealing for deep submicron crystal[40, 41]. However, recent experiment and simulation results $[6,42]$ suggest that it is relative difficult for bcc crystal to reach such kind of dislocation starvation trend. Generally, numbers of dislocations are left after deformation. This feature is also well reproduced by our simultions (see Fig. 7). Then, of special interest is the question whether most of the remaining dislocations are of a screw character similar to that in the bulk bcc crystal. To answer this question, some typical dislocation configurations corresponding to engineering strain $0.4 \%$ are given in Fig. 7. The screw dislocation is distinguished from mixed dislocations by using different colors. Here, one dislocation segment is recognized as a screw one, if the angle between its tangent direction and Burgers vector direction is smaller than $15^{\circ}$. Fig. 7 clearly shows how temperature and size influence the structure and amount of screw dislocations. In the case of low temperature and large size, numbers of elongated straight screw dislocations are observed and they dominate the plastic behaviour. In contrast, for small sample or under high temperature, only small amount of short screw dislocations are 

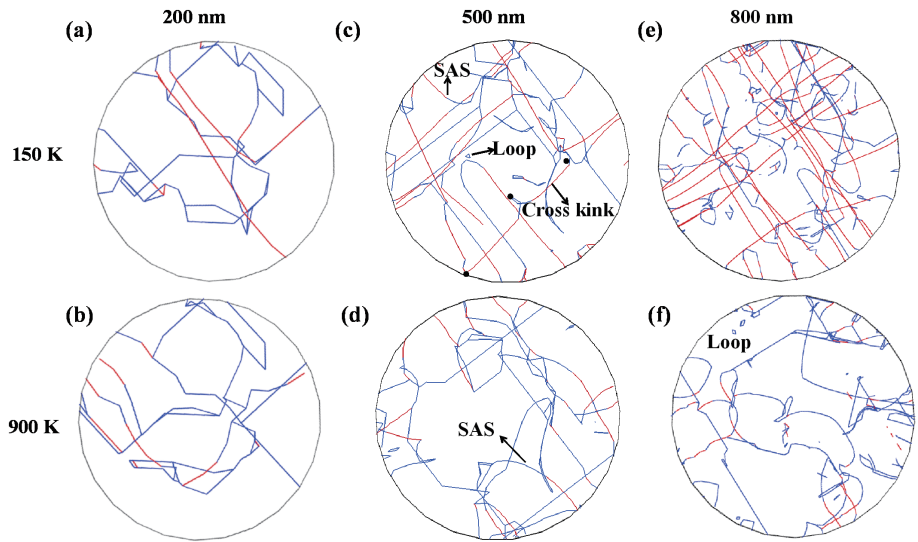

(d)

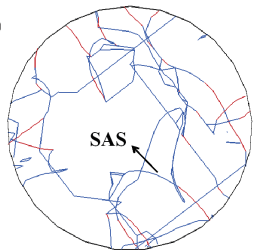

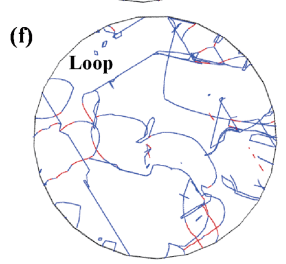

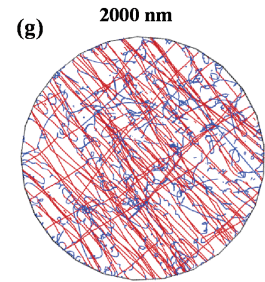

(h)

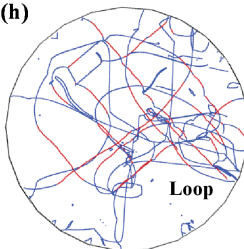

Figure 7: Dislocation configurations in micropillars with different sizes at different temperatures when the engineering strain is $0.4 \%$ from the top view. Screw dislocations are indicated in red, while mixed dislocations are in blue

To explore it further, the fraction of screw dislocation density among the total dislocation density is calculated. In order to give a sense to the statistic representation, the result for each diameter and temperature in Fig. 8 (a) is the average over several realizations with different initial dislocation configurations.

It clearly indicates that decreasing the sample size has the similar influence of increasing the temperature on decreasing the storage ability of screw dislocation. For clarity, the averaged percent value of screw dislocation density during the deformation stage $\varepsilon>0.2 \%$ is further evaluated and plotted in Fig. 8 (b). The dash dotted line represents the statistic value of the fraction of screw dislocation density if dislocations randomly orients in the slip plane, which is related to the screw dislocation definition and is calculated as $15^{\circ} \times 4 / 360^{\circ}$ here. Fig. 8 (b) demonstrates that the role of screw dislocation quickly become less important as the external size decreases even at low temperature. Similar phenomenon is also observed experimentally. Cheng et al. find that in nanocrystalline bcc Mo, mixed dislocations gradually becomes the dominated type of dislocation with reducing grain size[43].

This phenomenon can be further understood from two aspects. One is that 

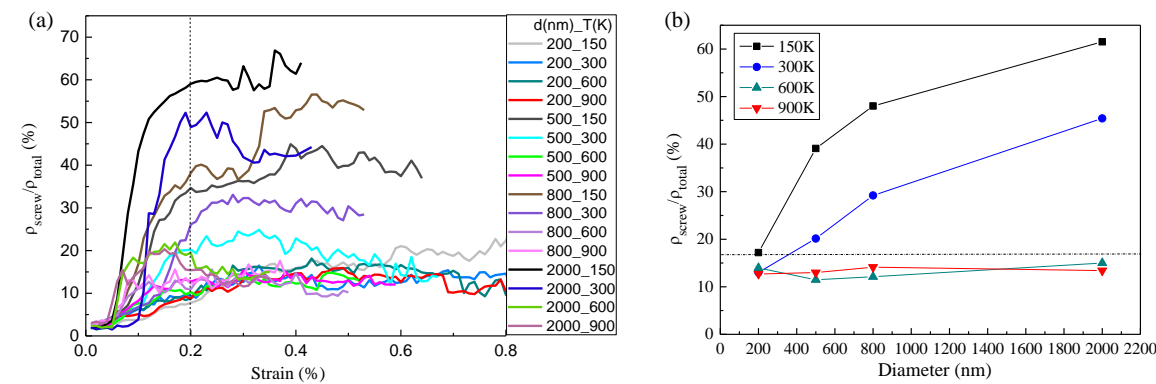

Figure 8: (a) Evolution of the percent of screw dislcation density for W micropillar with different sizes at different temperatures. The results are avaraged over multiple simulation realizations. (b)Avaraged percent of screw dislcation density during the deformation stage $\varepsilon>0.2 \%$

even though the screw dislocation at low temperature has low mobility, its lifetime is still short due to the limited glide free path at small scales. For example, some mixed dislocations may directly glide out of the crystal without forming long screw dislocations, or the screw dislocation is close to a free surface, and is easy to leave the crystal with the aid of the attractive image force. The other is that the mobility of screw dislocation is also strongly influenced by the high flow stress at small scales, which is analysed in detail in the following section.

\subsection{High stress enhances screw dislocation mobility}

In the case of low temperature, the movement of screw dislocation is always assisted by the thermally-activated kink-pair mechanism. As stress increases, less thermal energy is needed to overcome the Peierls potential[37, 44]. Physcially speaking, the increase of yield strength will contribute to the decrease of activation volume $V$ for the thermally activated kink pair nucleation [37, 45],

$$
\begin{aligned}
V & =b h \lambda^{*} \\
\lambda^{*} & =\left(\frac{\mu b h}{8 \pi \tau_{a}}\right)^{1 / 2}
\end{aligned}
$$

where $b$ is burgers vector, $h$ is kink height, $\lambda^{*}$ is the separated distance of kink pair, $\tau_{a}$ is applied resolved shear stress. The smaller the activation volume, 
the less the thermal energy the screw dislocation motion requires. Thus, if ative high strain rate. The strain rate sensitivity at submicron scales will be further investigated in our future work. 


\section{A dislocation mechanism map}

305

The analysis presented above suggests that, at small sample size, even when the test temperature is lower than the critical value, a mechanism transition from a kink-pair controlled motion to a phonon drag dominated motion will occur. This must happen, due to the limited external size and "the smaller is stronger" trend, as is schematically shown in Fig. 9. In the phonon drag dominated zone, the dislocation behavior is similar to that of FCC crystals. Accordingly, it is reasonable to propose that, with the reduction of the sample size, the dominant dislocation mechanism changes from dislocation forest hardening (with extensive dislocation-dislocation interactions), to a single-arm source mechanism as a result of surface truncation (see Fig. $7(\mathrm{~d})$ ). This is verified by the present simulation results of dislocation configuration evolution. Meanwhile, simulation results indicate that multiple dislocation sources can simultaneously operate at the same time in large samples, while only one dislocation source operates intermittently in small samples, leading to the occurrence of pronounced strain bursts. Note that the role of cross slip is more important in BCC crystals, because of the more available cross slip planes. Thus, many dislocation loops are formed after multiple cross slip events, as can be seen in Fig. 7(f) and (h).

In contrast, in the kink dominated regime, plastic deformation is dominated by the motion of screw dislocations, which is assisted by the formation of kinkpairs. Close examination of the simulation results illustrates several types of dislocation multiplication mechanisms, as indicated in Fig.10. The detailed evolution processes for each mechanism are schematically summarized in Fig.11. In Fig.11 (c), well-known dislocation sources induced by junction formation are shown, exhibiting a half-track-field-like geometry. The other types of dislocation multiplication mechanisms are assisted by the occurrence of cross-slip. Considering a long screw dislocation with the indicated Burgers vector, one cross-slip event occurs via kink pair nucleation on the cross-slip plane. If another kink pair nucleates on the original slip plane, and they migrate towards each other, a 


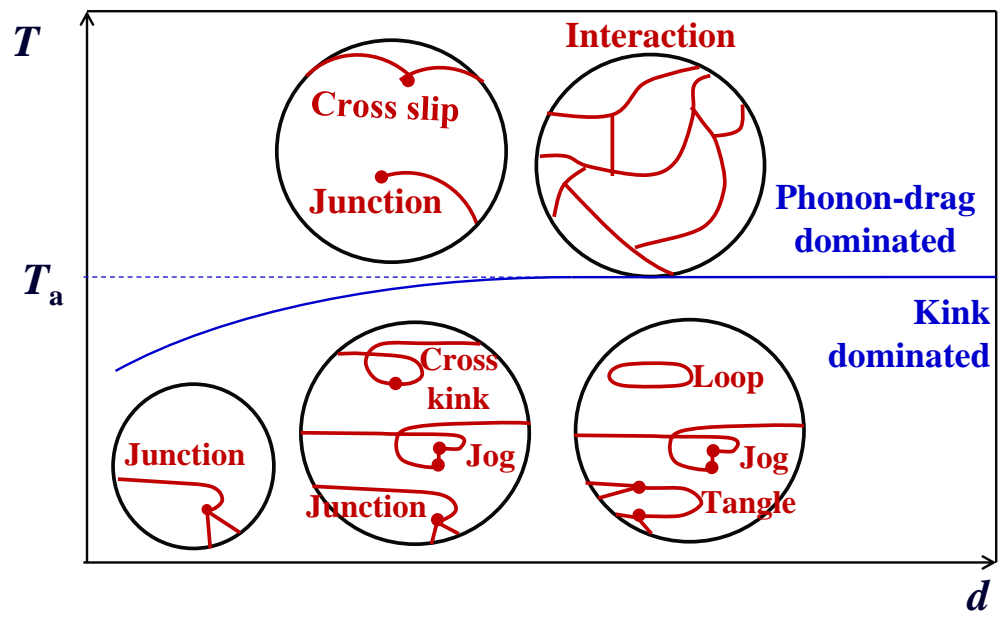

Figure 9: Dislocation mechanism map of BCC crystals, as a function of sample size and test temperature.

cross-kink forms[12], as shown in Fig.11 (b). In the current simulation, one dislocation segment is identified as a kink if it has the same Burgers vector and slip plane as its connected dislocation lines, but is much shorter than its connected long dislocation lines. The minimum length of the kink that can be resolved in the simulation is $40 b$, where $b$ is the magnitude of burgers vector. Note that the atomic sized kink mechanism is indirectly considered in the mobility law. The two arms of the cross-kink generally have different glide velocities, leading to the motion of their intersection point. In the case of low stress and limited size, this intersection point may annihilate at the free surface, as indicated in Fig.11 (d). However, if the stress is high enough, dislocation multiplication occurs due to the bowing out of the two arms (see Fig.11 (f)). This is also observed in previous molecular dynamics simulations [42]. This mechanism is termed "self multiplication", and is widely used to explain why dislocation starvation is seldom observed in small BCC samples [13]. Our simulation results suggest that dislocation jogs can also serve as active dislocation sources, and can induce significant dislocation multiplication. This connection between jogs and dislocation multiplication was also experimentally reported in $\mathrm{Nb}$ and Fe over a wide range 
of temperatures [48-50]. Such jogs are generally formed by multiple cross slip events (see Fig.11 (e)). The fate of dislocation segments around a jog depends on the jog length $y$ [51]. If the difference between the applied resolved shear stress $\tau_{a}$ and lattice friction stress $\tau_{f}$ is lower than the interaction stress between segments separated a distance $y$, namely: $\tau_{a}-\tau_{f}<0.25 \mu b /[2 \pi(1-\nu) y]$, these dislocation segments cannot pass each other. Then, dislocation dipoles form, as illustrated in Fig.11 (g). Otherwise, they operate independently, similar to single arm sources (SAS, see Fig.11 (i)). On the other hand, the occurrence of another cross-slip event will cause recombination between the two arms, giving rise to a closed prismatic loop. Therein, and as shown in Fig.11 (j) and (k), small loops are left in the sample as debris, while larger loops may expand as a new multiplication source driven by the applied stress.

(a) $d=200 \mathrm{~nm}$

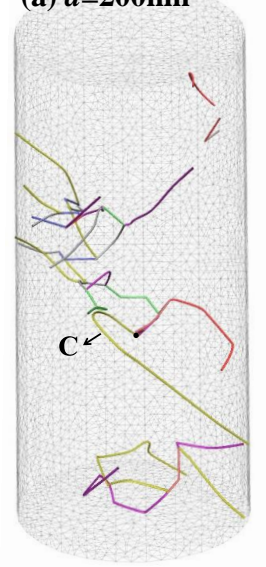

(b) $d=500 \mathrm{~nm}$

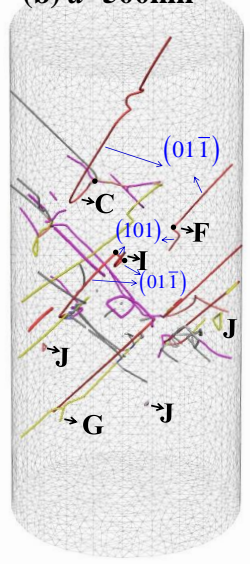

(c) $d=800 \mathrm{~nm}$

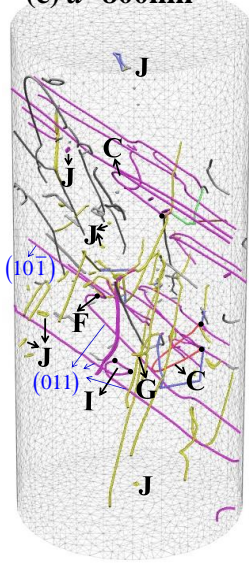

(d) $d=2000 \mathrm{~nm}$

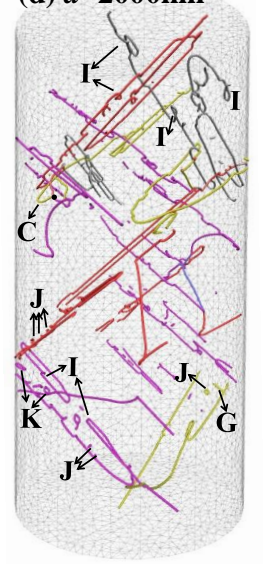

Figure 10: Typical dislocation configurations at $150 \mathrm{~K}$ when the applied stress is $1.1 \sim 1.2$ GPa for W micropillars with (a) d=200 nm, (b) d=500 nm, (c) d=800 nm and (d) d=2000 nm. Salient dislocation mechanisms are marked by letters, including $(\mathrm{C})$ pinning on a dislocation tangle, (F) cross kink multiplication, (I) pinning on super-jogs, (K) loop expansion, (G) dislocation dipole, and $(\mathrm{J})$ debris loops. Different colors in the figure are used to represent different Burgers vectors, grey $=(111)$, purple $=(1 \overline{1} 1)$, orange $=(11 \overline{1})$, red $=(1 \overline{11})$. Some of the blue numbers in (b) and (c) indicate the slip planes.

In all simulated micropillars, such as those typically shown in Fig.10, when 


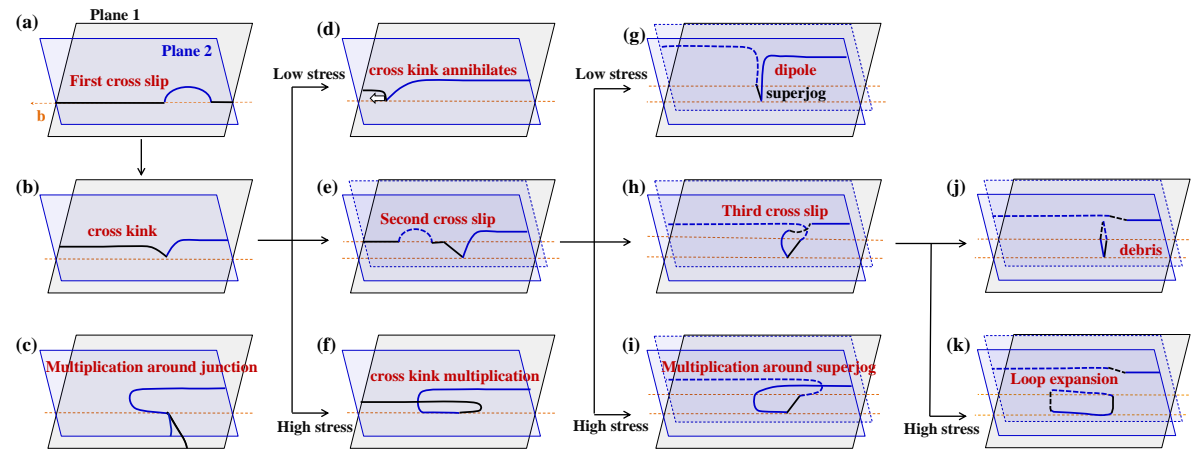

Figure 11: Schematic showing the mechanisms of dislocation multiplication, debris formation, and dipole formation in BCC crystals at low temperature. The black dislocation lines are in plane 1 and the blue ones are in plane 2

the diameter is less than $200 \mathrm{~nm}$, the most frequent observed multiplication mechanism is the single arm source around a dislocation junction. According to Fig.1, even though the initial dislocation density in the pillar with diameter $200 \mathrm{~nm}$ is much higher than that in larger pillar, the initial dislocation junction number is still relatively small. Thus, the reason that the dislocation junction mechanism is widely observed in small samples should not be artificially induced by the initial dislocation configuration. It can be understood in terms of the limited space available for multiple cross slip events to occur, which means lower possibility of forming and activating the source of cross kink and super-jogs. For larger diameters of $500 \mathrm{~nm}$ and $800 \mathrm{~nm}$, however, the cross kink multiplication and jog-induced multiplication mechanisms are also widely observed. This is on the one hand related to the pronounced surface-induced image force, which can promote the occurrence of cross slip and the formation of cross kinks[42], and on the other hand related to the high stress levels and relative large space that can accomodate dislocation multiplication. For larger samples with diameter $2000 \mathrm{~nm}$, during the initial deformation stage, the dominant dislocation sources are double-pinned half-loop dislocations, such as those schematically shown in Fig. 9. After many long screw dislocations are left inside the pillar due to their slow mobility, both multiple available cross slip planes and complex dislocation 

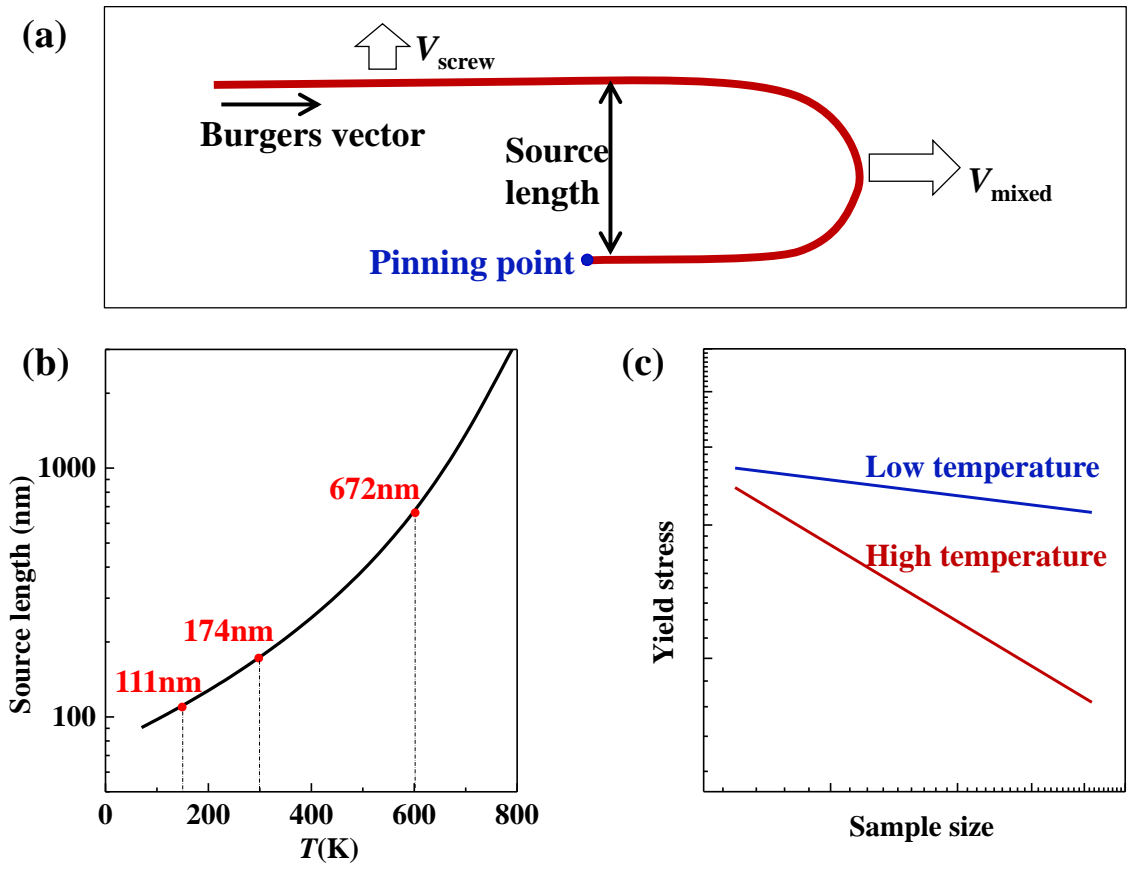

Figure 12: (a) Schematic showing the common source in BCC crystals at low temperatures. The pinning point can originate from a junction, cross-kink, or a jog; (b) The critical length of the source that can be activated in bulk BCC crystals at different temperatures; (c) Schematic illustrating the influence of sample size at different temperatures in a logarithmic manner.

interactions favor the occurrence of cross slip, leading to the formation of a large density of superjogs. Thus, the jogged screw dislocation is widely observed in the following plastic flow stage, as is evidenced in Fig.10(d). In addition, even for larger samples, the flow stress at relatively low temperatures is also high, which can easily reach the criterion for dislocation multiplication around jogs. Therefore, the most frequently observed multiplication mechanism becomes the jog-induced multiplication process. This observation is also consistent with previous DDD simulation results [52], and with experimental observations [50]. Dislocation multiplication induced by loop expansion leaving plenty of dislocation debris is also observed in our simulations (such as Fig.10 and Fig. 7(c),(e) 
and $(\mathrm{g})$ ). For larger micropillars, the motion of jogged screw dislocations results in a high density of dislocation debris, which explains why plenty of prismatic debris loops are observed in bulk $\mathrm{W}$ crystals[53].

Based on the analysis above, a dislocation mechanism map is presented in Fig. 9, summarizing the interplay between the sample size and its temperature in determining the source operative mechanism. The map clearly shows that both temperature and size cooperate to induce transitions between dominant dislocation mechanisms. To understand the contribution of a given mechanism transition on the extent of its temperature dependence, we consider the following argument. For a specific temperature, the size effect can be attributed to its influence on the dislocation source operation, which can be mainly understood from two aspects. One is the truncation effect on the source length, and the other is the limited available number of dislocation sources. In the following, analysis is first given from these two perspectives to show that the size effect is indeed weaker in the low temperature regime.

First, in the kink dominated regime, even though multiple dislocation mechanisms may exist, a common dislocation source character can be observed for different characteristic sizes, as plotted in Fig.12(a). Here, the pinning point may originate from a junction, cross kink, or a jog. Irrespective of these differences, the operation stress of such kind of source can be roughly estimated according to the line tension model, namely $\tau_{a}-\tau_{f}=\mu b / \lambda$, where $\lambda$ is its critical length, as indicated in Fig.12 (a). Since the flow stress in bulk bcc is high ${ }_{415}$ at low temperatures, the critical source length can reach a small value. Fig. 12 (b) give a crude estimation of the source length that can be activated in bulk W sample, according to the fitted experimental flow stress data given by the black solid line in Fig. 6. It can be seen that when the temperature is lower than room temperature, even the bulk flow stress can activate sources with length smaller than $174 \mathrm{~nm}$. Therefore, for the considered size regime in this investigation, the truncation effect induced by the external size on the source length is weaker at lower temperature.

Second, the number of pinning points is generally limited in small samples. 
However, at low temperatures, in addition to pinning points induced by junctions, both cross-kinks and jogs can supply pinning points. Thus, the dislocation source density is larger in this case. The present analysis is the first to clearly establish an understanding of the experimental observations that the influence of sample size on plastic flow in BCC crystals is weak at low temperatures [17] from the viewpoint of dislocation mechanism. The trend in this anomalous size dependence of the flow stress on temperature is schematically illustrated in Fig.12 (c). Considering that the power law trend is always observed for size dependence, the two straight lines are plotted in the logarithmic schematic diagram. Fig.12 (c) clearly shows that the weaker size dependence at lower temperatures can exactly explain the weaker temperature effect at smaller scales.

\section{6. Summary and Conclusions}

Atomistically informed dislocation dynamics simulations of the tension tests of $\mathrm{W}$ single-crystal micropillars have been systematically carried out. The aim was to map out the combined influence of sample size and test temperature on the flow stress and operative dislocation mechanisms. The simulation results show general good agreement with available experimental data on the flow stress dependence on size and temperature, and with the experimental observations of dislocation microstructures in W. Contrary to the popular notion that bcc crystals generally have strong temperature dependence of the flow stress, in the present work we show a gradual vanishing temperature sensitivity of the flow stress as the sample size decreases. Careful analysis of the simulation results suggests that this is related to the weak role of screw dislocations at small scales, and the inability of small samples to store them. The fraction of screw dislocations decreases sharply with the reduction of sample size, even at low temperatures. At the same time, the very high flow stress in small scale bcc crystals, together with the image force effect, can greatly enhance the mobility of screw dislocations. This suggests that the mixed dislocations control the plastic response in bcc metals in the very small scale regime as against bulk 
specimens. These can further result in a transition of the dislocation mobility mechanism from kink-pair dominated to phonon-drag dominated, even when the temperature is lower than the traditional critical temperature. The critical temperature for this mobility mechanism transition has been found to depend on sample size, decreasing as the size gets smaller. In addition, various dislocation multiplication mechanisms in submicron bcc crystals have been discovered. At low temperatures and small sample size, the dominant mechanisms have been found to be junction formation, cross-kink formation, and jog formation. As the size increases further, still at low temperatures, dislocation loop expansion and tangles become dominant. At temperatures above the critical value, the cross-slip mechanism participates in small sizes together with junctions, transitioning to dislocation tangles and interaction dominated behavior at larger sample sizes.

\section{Acknowledgements}

This material is based upon work supported by the U.S. Department of Energy, Office of Science, Office of FusionEnergy Sciences, under Award Number DE-FG02-03ER54708, and the US Air Force Office of Scientific Research

470 (AFOSR), under award number FA9550-11-1-0282. We would like to thank Prof. Jaime Marian and Prof. Jonathan A. Zimmerman for inspiring comments and discussions.

\section{References}

[1] A. Argon, S. Maloof, Plastic deformation of tungsten single crystals at low temperatures, Acta Metall. 14 (1966) 1449-1462.

[2] R. Schnitzel, Deformation of tungsten single crystals from- $77^{\circ} \mathrm{c}$ to $800^{\circ} \mathrm{c}$, J. Less Common Metals 8 (1965) 81-89.

[3] H. Conrad, W. Hayes, Correlation of the thermal component of the yield stress of the bcc metals, Am. Soc. Metals, Trans. Quart. 56 (1963). 
[4] M. Rieth, J. Boutard, S. Dudarev, T. Ahlgren, S. Antusch, N. Baluc, M.-F. Barthe, C. Becquart, L. Ciupinski, J. Correia, et al., Review on the EFDA programme on tungsten materials technology and science, J. Nucl. Mater. 417 (2011) 463-467.

[5] M. D. Uchic, P. A. Shade, D. M. Dimiduk, Plasticity of micrometer-scale single crystals in compression, Annu. Rev. Mater. Res. 39 (2009) 361-386.

[6] J.-Y. Kim, D. Jang, J. R. Greer, Tensile and compressive behavior of tungsten, molybdenum, tantalum and niobium at the nanoscale, Acta Mater. 58 (2010) 2355-2363.

[7] M. Zaiser, J. Schwerdtfeger, A. Schneider, C. Frick, B. G. Clark, P. Gruber, E. Arzt, Strain bursts in plastically deforming molybdenum micro-and nanopillars, Philos. Mag. 88 (2008) 3861-3874.

[8] D. Caillard, J.-L. Martin, Thermally activated mechanisms in crystal plasticity, volume 8, Elsevier, 2003.

[9] R. Gröger, A. Bailey, V. Vitek, Multiscale modeling of plastic deformation of molybdenum and tungsten: I. atomistic studies of the core structure and glide of $1 / 2<111>$ screw dislocations at 0k, Acta Mater. 56 (2008) 5401-5411.

[10] S. Brinckmann, J.-Y. Kim, J. R. Greer, Fundamental differences in mechanical behavior between two types of crystals at the nanoscale, Phys. Rev. Lett. 100 (2008) 155502.

[11] L. Zhang, T. Ohmura, K. Sekido, T. Hara, K. Nakajima, K. Tsuzaki, Dislocation character transition and related mechanical response in a bodycentered cubic single crystal, Scripta Mater. 67 (2012) 388-391.

[12] C. Marichal, K. Srivastava, D. Weygand, S. Van Petegem, D. Grolimund,

P. Gumbsch, H. Van Swygenhoven, Origin of anomalous slip in tungsten, Physical review letters 113 (2014) 025501. 
[13] L. Huang, Q.-J. Li, Z.-W. Shan, J. Li, J. Sun, E. Ma, A new regime for mechanical annealing and strong sample-size strengthening in body centred cubic molybdenum, Nat. Commun. 2 (2011) 547.

[19] G. Po, M. S. Mohamed, T. Crosby, C. Erel, A. El-Azab, N. Ghoniem, Recent progress in discrete dislocation dynamics and its applications to micro plasticity, JOM 66 (2014) 2108-2120.

[20] Y. Cui, Z. Liu, Z. Zhuang, Theoretical and numerical investigations on 530 confined plasticity in micropillars, J. Mech. Phys. Solids 76 (2015) 127143.

[21] Y. Cui, Z. Liu, Z. Zhuang, Quantitative investigations on dislocation based discrete-continuous model of crystal plasticity at submicron scale, Int. J. Plasticity 69 (2015) 54-72. 
[22] J. A. El-Awady, Unravelling the physics of size-dependent dislocationmediated plasticity, Nat. Commun. 6 (2015).

[23] M. Tang, L. Kubin, G. Canova, Dislocation mobility and the mechanical response of bcc single crystals: a mesoscopic approach, Acta Materialia 46 (1998) 3221-3235.

[24] J. Chaussidon, C. Robertson, D. Rodney, M. Fivel, Dislocation dynamics simulations of plasticity in fe laths at low temperature, Acta Mater. 56 (2008) 5466-5476.

[25] Z. Wang, I. Beyerlein, An atomistically-informed dislocation dynamics model for the plastic anisotropy and tension-compression asymmetry of bcc metals, Int. J. Plasticity 27 (2011) 1471-1484.

[26] K. Srivastava, R. Gröger, D. Weygand, P. Gumbsch, Dislocation motion in tungsten: atomistic input to discrete dislocation simulations, Int. J. Plasticity 47 (2013) 126-142.

[27] G. Po, Y. Cui, D. Rivera, D. Cereceda, J. Marian, N. Ghoniem, A dislocation mobility law for bcc metals with applications to tungsten micro pillar deformation, Acta mater. (to be submitted).

[28] N. Ghoniem, M, S.-H. Tong, L. Sun, Parametric dislocation dynamics: a thermodynamics-based approach to investigations of mesoscopic plastic deformation, Phys. Rev. B 61 (2000) 913.

[29] G. Po, M. Lazar, D. Seif, N. Ghoniem, Singularity-free dislocation dynamics with strain gradient elasticity, J. Mech. Phys. Solids 68 (2014) 161-178.

[30] J. D. Eshelby, The determination of the elastic field of an ellipsoidal inclusion, and related problems, in: Proceedings of the Royal Society of London A: Mathematical, Physical and Engineering Sciences, volume 241, The Royal Society, 1957, pp. 376-396. 
[31] G. Po, N. Ghoniem, Mechanics of defect evolution library, model, 〈https://bitbucket.org/model/model/wiki/home〉 (2015).

[32] W. Kocks, Thermodynamics and kinetics of slip, Progr. Mater. Sci. 19 (1975) 291.

[33] R. Gröger, V. Racherla, J. Bassani, V. Vitek, Multiscale modeling of plastic deformation of molybdenum and tungsten: Ii. yield criterion for single crystals based on atomistic studies of glide of $1 / 2<111>$ screw dislocations, Acta Mater. 56 (2008) 5412-5425.

[34] A. Stukowski, D. Cereceda, T. D. Swinburne, J. Marian, Thermallyactivated non-schmid glide of screw dislocations in w using atomisticallyinformed kinetic monte carlo simulations, International Journal of Plasticity 65 (2015) 108-130.

[35] D. Cereceda, M. Diehl, F. Roters, D. Raabe, J. M. Perlado, J. Marian, Unraveling the temperature dependence of the yield strength in singlecrystal tungsten using atomistically-informed crystal plasticity calculations, International Journal of Plasticity (2015).

[36] Y. Kamimura, K. Edagawa, S. Takeuchi, Experimental evaluation of the peierls stresses in a variety of crystals and their relation to the crystal structure, Acta Materialia 61 (2013) 294-309.

[37] A. Schneider, C. Frick, B. Clark, P. Gruber, E. Arzt, Influence of orientation on the size effect in bcc pillars with different critical temperatures, Mater. Sci. Eng. A 528 (2011) 1540-1547.

[38] A. Schneider, B. Clark, C. Frick, P. Gruber, E. Arzt, Effect of orientation and loading rate on compression behavior of small-scale mo pillars, Mater. Sci. Eng. A 508 (2009) 241-246.

[39] A. Schneider, D. Kaufmann, B. Clark, C. Frick, P. Gruber, R. Mönig, O. Kraft, E. Arzt, Correlation between critical temperature and strength of small-scale bcc pillars, Phys. Rev. Lett. 103 (2009) 105501. 
[40] Z. Shan, R. K. Mishra, S. S. Asif, O. L. Warren, A. M. Minor, Mechanical annealing and source-limited deformation in submicrometre-diameter ni crystals, Nat. Mater. 7 (2008) 115-119.

[41] J. R. Greer, W. D. Nix, Nanoscale gold pillars strengthened through dislocation starvation, Phys. Rev. B 73 (2006) 245410.

[42] C. R. Weinberger, W. Cai, Surface-controlled dislocation multiplication in metal micropillars, Proc. Natl. Acad. Sci. U.S.A. 105 (2008) 14304-14307.

[43] G. Cheng, W. Jian, W. Xu, H. Yuan, P. Millett, Y. Zhu, Grain size effect on deformation mechanisms of nanocrystalline bcc metals, Mater. Res. Lett. 1 (2013) 26-31.

[44] A. Ngan, M. Wen, Atomistic simulation of energetics of motion of screw dislocations in bcc fe at finite temperatures, Computational materials science 23 (2002) 139-145.

[45] A. Seeger, P. Schiller, The formation and diffusion of kinks as the fundamental process of dislocation movement in internal friction measurements, Acta Metall. 10 (1962) 348-357.

[46] J. R. Stephens, Dislocation structures in single-crystal tungsten and tungsten alloys, Metall. Mater. Trans. 1 (1970) 1293-1301.

[47] R. Huang, Q.-J. Li, Z.-J. Wang, L. Huang, J. Li, E. Ma, Z.-W. Shan, Flow stress in submicron bcc iron single crystals: Sample-size-dependent strainrate sensitivity and rate-dependent size strengthening, Mater. Res. Lett. (2015) 1-7.

[48] F. Louchet, L. Kubin, D. Vesely, In situ deformation of bcc crystals at low temperatures in a high-voltage electron microscope dislocation mechanisms and strain-rate equation, Philos. Mag. A 39 (1979) 433-454.

[49] F. Louchet, L. Kubin, Dislocation processes in bcc metals, Phys. Status. Solidi. A $56 \quad$ (1979) $169-176$ and http://www.numodis.fr/tridis/TEM/mechanisms/multiplication.html. 
[50] J. Low, A. Turkalo, Slip band structure and dislocation multiplication in silicon-iron crystals, Acta metall. 10 (1962) 215-227.

[51] W. Johnston, J. Gilman, Dislocation multiplication in lithium fluoride crystals, J. Appl. Phys. 31 (1960) 632-643.

[52] M. Rhee, D. H. Lassila, V. V. Bulatov, L. Hsiung, T. D. de la Rubia, Dislocation multiplication in bcc molybdenum: a dislocation dynamics simulation, Philos. Mag. Lett. 81 (2001) 595-605.

[53] A. S. Argon, Strengthening mechanisms in crystal plasticity, Oxford University Press Oxford, 2008. 
Graphical Abstract

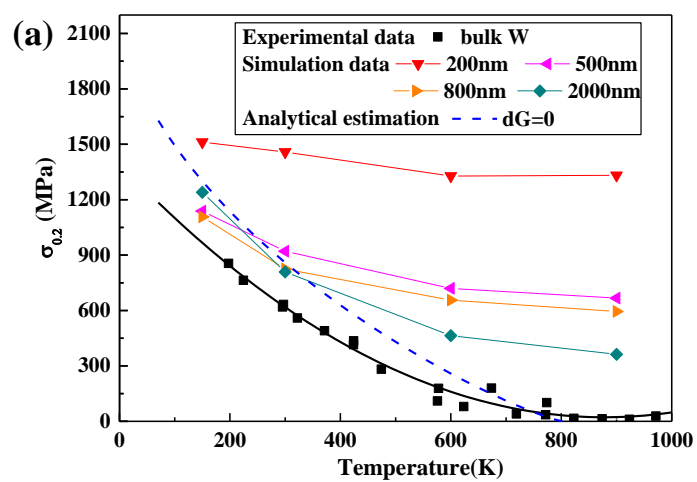

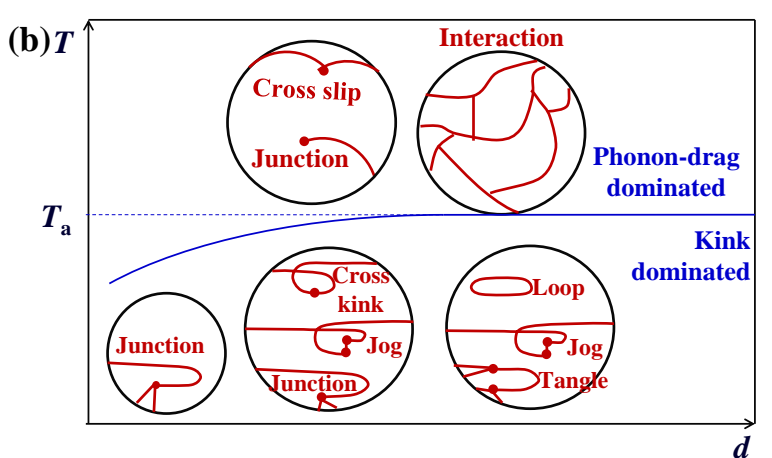

